Monatsschrift f. Geburtshülfe u. Gynäkologie 1924;67:123-124

\title{
IV. Buchbesprechungen
}

Halban-Seitz, Biologie und Pathologie des Weíbes. 7. Lieferung.

F. Kermauner, Fehlbildungen der weiblichen Geschlechtsorgane, des Harnapparates und der Kloáke. Fragliches Geschlecht. - K. Reifîerscheidt, Lage- und Geslaltsveränderungen der weiblichen Genilalorgane (Anhang). Kermauners Beitrag ist in seiner voll ausschöpfenden Gründlichkeit, in der anziehend anregenden Art der Darstellung, welche durch eine Fülle von Abbiidungen ergänzt wird, ein leuchtendes Vorbild eines Handbuchartikels. Er gehört unbestritten zu den besten des großzügigen Werkes! Der Leser wird von Anfang bis zu Ende festgehalten und nimmt den Eindruck als Gewinn mit sich fort, dieses Kapitel in alien seinen vielfältigen Einzelheiten kennen gelernt zu haben. Ref. hat vielfach einzelne Kapitel wiederholt durchgelesen und reiche Belehrung dankbar entgegengenommen, in Genese, Anatomie, Klinik und Therapie der uns oft so rätselhaft entgegentretenden Genital-Fehlbildungen.

Reifferscheidt bringt in diesem ersten Teíl die einschlägigen Verlagerungen des Uterus und den Umfang der Darstellung des Genitalprolaps. Zahlreiche Abbiidungen aus unserer reichen Literatur geben der Diktion dankenswerte Klarheit. Der Leser sieht mit Interesse dem Abschluß entgegen. A. Martin.

124 Literaturverzeichnis.

8. Lieferung. Stickel, Vor- und Nachbehandlung gynäkologischer Operaíionen. Thaler, Allgemeinnarkose und Lokalanästhesie in Geburtshilfe und Gynäko-logie.

Stickel gibt in kurzen lapidaren Sätzen die heute geltenden Vor-schriften fur die Anti- und Asepsis in der Gynäkologie der Klinik von Franz. Es ist sehr wohlgetan, die einzelnen Stufen der Vorbereitungen der Patientinnen, des Operationsraumes, der Instrumente und Verband-stoffe in solcher Form zusammenzustellen und den Operationsverlauf. Bei der Nachbehandlung finden Ileus und Peritonitis, Schmerzbekämpfung und Fieberbehandlung, auch Haut- und Mundpflege kurze Erörterung; auch die Verhütung der postoperativen Thromboembolie. Die einschlägige Literatur wird auf 12 Seiten angeführt. Ref. mòchte die Frage aufwerfen, ob eine solehe Ausführlichkeit im Plan des Gesamtwerkes liegt ? Sie wird in den einzelnen Abschnitten verschieden beantwortet. Dem Ref. erscheint sie „handbuch”-gemäß. Nach vielseitiger neuerer Auffassung genügt es, die letzten ausführlichen Arbeiten mit ihrer speziellen Literaturzusammen-stellung zu nennen, im Interesse der Ausdehnung und des Preises des Buches.

Thaler gibt eine zusammenfassende Übersicht über die in Geburtshilfe und Gynäkologie zur Anwendung gelangenden Methoden der Allgemeinnarkose, deren Technik, Gefahren und Gefahrenverhütung. Es ware dankenswert, wenn dabei der Preis der einzelnen Verfahren erwähnt würde, eii»o Angelegenheit, welche immerhin in der Praxis eine gewisse Bedeutung hat. - Es folgt eine ausführliche Erörterung der Anwendung der Inhalationsnarkose. Th. tritt dabei für 
die hauptberufsmäßige Tätig-keit eines Arztes statt der Hilfspersonen ein, ein Fortschritt, der sich hauptsächlich in den Vereinigten Staaten durchgesetzt hat. Der Dämmer-schlaf der Gebärenden ist nach Th.s Auffassung eine Domäne der An-stalten, eine Auffassung, der sich Ref. anschließt.

Der Abschnitt über Lokalanästhesie gibt eine recht lesonswerte Darstellung der vielfältigen Varianten des Verfahrens. Th. bezeichnet die Erfolgsaussichten der parametramen Infiltrationsanästhesie bei alien vaginalen Bauchhöhlenoperationen als sehr günstig. A. Martin.

V. Literaturverzeichnis.

Geburtshülfe.

Aschner, Die Konstitution der Frau und ihre Beziehungen zur Geburtshilfe und Gynäkologie. Bd. 1. Allgemeine Konstitutionslehre. München 1924. J. F. Bergmann.

Baumm, Zur künstlichen Atmung bei Asphyxia neonat. Z. f. G. Nr. 17. S. 917.

Bier, Immunität durch Befruchtung. Munch, med. Woch. Nr. 16. S. 491.

Böttner, Experimentelle und klinische Untersuchungen zur Frage: Blut-transfusion (Zitratblut) und Anaphylaxie. Dtsch. med. Woch. Nr. 10. S. 599.

Bown, Rößlins Rosengarten der schwangern Frauen und Hebammen. Bespr. m. Abb. Surg. Gyn. obstet. April 24. S. 570. 